OPEN ACCESS

Edited by:

Bo Yang,

Kunming University of Science and

Technology, China

Reviewed by:

Xiaomeng $A i$

Huazhong University of Science and

Technology, China

Binyu Xiong,

Wuhan University of Technology,

China

*Correspondence:

Junhui Li

lijunhui@neepu.edu.cn

Specialty section:

This article was submitted to

Smart Grids,

a section of the journal

Frontiers in Energy Research

Received: 10 July 2021

Accepted: 02 August 2021

Published: 23 September 2021

Citation:

Li C, Zhang Z, Li J, Ma Y and Zou J (2021) Design of Control Strategy and Effect Evaluation for Primary Frequency Regulation of Wind Storage System.

Front. Energy Res. 9:739439.

doi: 10.3389/fenrg.2021.739439

\section{Design of Control Strategy and Effect Evaluation for Primary Frequency Regulation of Wind Storage System}

\author{
Cuiping $L i^{1}$, Zheshen Zhang ${ }^{2}$, Junhui $L^{1}{ }^{1 *}$, Yunbao $\mathrm{Ma}^{3}$ and Jiajun $\mathrm{Zou}^{4}$ \\ ${ }^{1}$ Key Laboratory of Modern Power System Simulation and Control and Renewable Energy Technology, Ministry of Education \\ (Northeast Electric Power University), Jilin, China, ${ }^{2}$ Wenzhou Power Supply Company, State Grid Zhejiang Electric Power \\ Company, Wenzhou, China, ${ }^{3}$ Zaozhuang Power Supply Company, State Grid Shandong Electric Power Company, Zaozhuang, \\ China, ${ }^{4}$ Jilin Province Electric Power Company of National Grid, Changchun, China
}

Because of wind sources' volatility and intermittent news, wind power has made a more and more heavy burden on the power system. This paper analyzes in detail the traditional control method, parallel control strategy and serial control strategy of the wind storage system, and combines the advantages of the two to propose an optimal control strategy. A comprehensive performance evaluation method for the primary frequency regulation of the ESS participating in the power grid is proposed based on the power system operation requirements. In the example, the frequency modulation performance of the optimal control strategy is verified by the evaluation method described in this paper in the Chinese frequency adjustment market.

Keywords: wind power, energy storage system, primary frequency regulation, control strategy, effect evaluation

\section{INTRODUCTION}

The total installed capacity of wind power in China is rising. As of August 2019, the total installed capacity of wind power in China has reached 198 million kilowatts (Shen et al., 2018; Yan and Zhao, 2018; Wind power grid-connected, 2019). Wind power itself has the unfavorable characteristics of large fluctuation and intermittent power output (Ramtharan et al., 2007; Teninge et al., 2009; Muyeen et al., 2012; Zertek et al., 2012; Dang et al., 2014; Luo et al., 2015; Peng et al., 2015), After wind power is connected to the grid, it will cause a large burden of frequency modulation on the grid. This burden may cause the power system to collapse (Li et al., 2018; Zhang et al., 2018; Annamraju and Nandiraju, 2019; Ayyarao and Ayyarao, 2019; Li et al., 2021). Many studies have been carried out the researches on the influence of wind power on power system frequency.

The following articles mainly solve the problem by improving the frequency regulation capability of wind turbines: In (Sun et al., 2018; Xing et al., 2018), since the wind turbine has no inertia, a control method for reducing the load of the wind turbine is proposed. To increase the reserve capacity of the frequency modulation system, the load capacity of the wind turbines is reduced. In (Wu et al., 2013; Tan et al., 2016), To solve the problem of insufficient capacity of wind turbine frequency modulation reserve, a method for tracking the power curve of suboptimal wind turbines is proposed. This method guarantees the ability of wind power to participate in frequency modulation by withdrawing from the MPPT mode of the wind turbine. But this method reduces the economy of wind turbines. In summary, the wind turbine's control method for frequency modulation is mainly at the reduced economy or load capacity of wind turbines. Due to its characteristics, the response speed of wind turbines is difficult to meet the needs of power system primary frequency modulation. 
The capacity of ESS can be configured as needed, and at the same time, it responds extremely fast to the demand for frequency modulation (Miao et al., 2015; Hu et al., 2016; Li et al., 2017; Tariq et al., 2018; Wu et al., 2018; Pandzic and Bobanac, 2019; Zhao et al., 2019). ESS can assist wind turbines to solve the problem of frequency modulation.

In the related literature of ESS assisting wind turbine to solve the problem of frequency modulation, in (Jing et al., 2005; Sun et al., 2019), the method of adjusting the frequency in stages solves the problem of economic decline caused by using too much energy storage, and uses the ESS to solve the part of the power system where the frequency changes rapidly. In (Hu et al., 2014), the frequency section control method of wind turbine and the ESS is proposed. This method mainly uses Fourier transform to distribute the high frequency components to the ESS and the remaining components to the wind turbine. In (Yan et al., 2016; Zheng et al., 2017), use the ESS to assist the wind turbine to improve the inertial response capability of the wind turbine, thereby improving the overall frequency regulation capability of the wind storage system.

In order to improve the frequency modulation performance, the above literature is mainly to improve the capability of wind turbine's frequency modulation or use energy storage to improve the frequency modulation performance of the wind turbine. But does not consider how to allocate to the wind turbine and ESS when the frequency modulation instruction is issued. Therefore, this paper proposes an optimal control method based on series control and parallel control based on the related issues of ESS auxiliary wind turbines participating in the primary frequency regulation of the power system. This method can take into account both economical and frequency regulation performance. The effectiveness of the above strategy is verified through simulation cases. Finally, a method is proposed for evaluating the performance of the ESS participating in the primary frequency modulation. The results prove that the optimized control method has the best comprehensive performance in the context of the frequency modulation market in China.

\section{ESTABLISHMENT OF MATHEMATICAL MODEL RELATED TO FREQUENCY MODULATION}

In this chapter, the models of traditional generating units participating in the power system, such as hydropower units and thermal power units, use commonly used traditional models (Kundur, 2001).

Because the selection of relevant parameters of power system frequency modulation is more complicated, and many parameters are empirical parameters, this paper chooses the parameters in documents (Miao et al., 2015) and (Kundur, 2001) as the main source of this paper.

The specific mathematical model is as follows.

\section{Mathematical Model of Wind Turbine}

At present, the mainstream wind turbines have zero inertia characteristics, so it is necessary to adopt certain technical means to make the wind turbines have the ability of conventional primary frequency modulation. And the main control methods of wind turbine are "virtual inertia control" and "pitch control." Both of them simulate the frequency response of traditional units in power system by changing the active output of wind turbines. The transfer function to build the virtual inertia is shown below:

$$
G(s)=\frac{\Delta P_{\text {wind } 1}}{\Delta f}=-\frac{k_{v d} s}{1+T_{\text {wind } 1} s}
$$

where $k_{v d}$ is the virtual inertia constant of the wind turbine, which is usually $8 ; T_{\text {wind } 1}$ is the inertial response time constant of the wind turbine, and the value is usually $0.1 \mathrm{~s}$.

The Transfer Function Formula for Pitch Control is as Follows

$$
G(s)=\frac{\Delta P_{\text {wind } 2}}{\Delta f}=-\frac{k_{\text {change }}}{1+T_{\text {wind } 2} s}
$$

where $k_{\text {change }}$ Is the frequency modulation coefficient for pitch control, usually 20; $T_{\text {wind }}$ is the pitch control time constant of the wind turbine, $3 \mathrm{~s}$.

In summary, the overall frequency response transfer function formula of the wind turbine is as follows:

$$
G_{w}(s)=-\frac{\left(k_{v d} T_{\text {wind } 2}\right) s^{2}+\left(k_{\text {change }} T_{\text {wind } 1}+k_{v d}\right) s+k_{\text {change }}}{1+\left(T_{\text {wind } 2}+T_{\text {wind } 1}\right) s+T_{\text {wind } 2} T_{\text {wind } 1} s^{2}}
$$

\section{Mathematical Model of the ESS}

The advantage of the ESS is that its capacity can be configured according to demand, and at the same time can respond to the fast-changing frequency modulation needs. Configuring the ESSs in wind farms can alleviate the pressure of frequency modulation of power systems. Since the ESS is deployed in the wind farm, the inertial response coefficient and the primary frequency modulation coefficient of the ESS are the same as the wind generator. The mathematical model of the ESS is as follows:

$$
G_{e}(s)=\frac{\Delta P_{\text {Energy }}(s)}{\Delta f(s)}=-\frac{k_{v d} s+k_{\text {change }}}{T_{\text {Energy }} s+1}
$$

where $\mathrm{T}_{\text {Energy }}$ is the frequency response time constant of ESS, generally $0.3 \mathrm{~s}$.

\section{CONTROL STRATEGY DESIGN}

After the power system sends down frequency modulation instructions, the traditional unit and wind storage system will allocate frequency modulation tasks according to how many frequency modulation problems are caused. The specific distribution method is as follows:

$$
\begin{aligned}
\Delta P_{\text {tra }} & =(1-p) * P_{\text {all }} \\
\Delta P_{w+e} & =p * P_{\text {all }}
\end{aligned}
$$




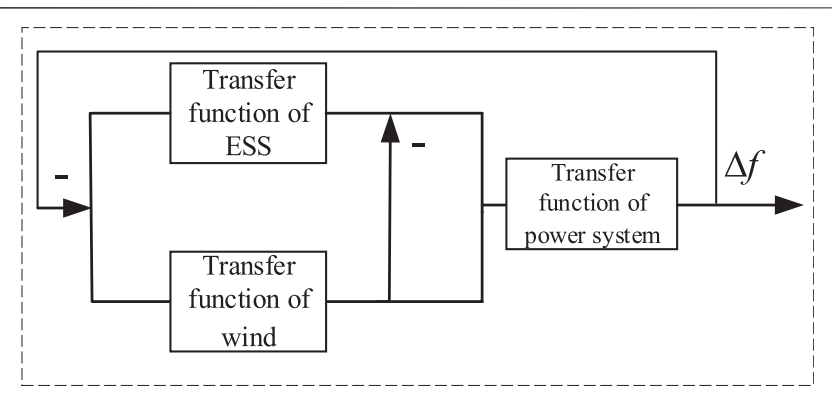

FIGURE 1 | Schematic diagram of wind storage system adopting serial control.

where $\Delta P_{w+e}$ is primary frequency regulation command responded by the wind storage system, unit $\mathrm{MW} ; \Delta P_{\text {tra }}$ is frequency modulation command that the traditional unit responds to, unit $\mathrm{MW}$; $\mathrm{p}$ is the proportion of wind power installed capacity in all units participating in frequency modulation, $0.2 ; \mathrm{P}_{\text {all }}$ is the total frequency modulation command issued by the power system.

In traditional frequency modulation reserve resources, thermal power units and hydropower units are allocated in the following ways.

$$
\begin{aligned}
\Delta P_{f} & =k * \Delta P_{\text {tra }} \\
\Delta P_{\text {wat }} & =(1-k) * \Delta P_{\text {tra }}
\end{aligned}
$$

where $\Delta \mathrm{P}_{\text {wat }}$ and $\Delta \mathrm{P}_{\mathrm{f}}$ are the regulators of hydroelectric units and thermal power units, respectively. $\mathrm{k}$ is the proportion of thermal power units, 0.8 .

\section{Control Strategy of Wind-Storage System}

The wind turbine and the ESS can be divided into three control modes according to the task assignment when receiving the frequency modulation instruction: serial mode, parallel mode, and optimal operation mode.

\section{Serial Control Mode of Wind-Storage System}

Serial control is a classic control strategy of wind/storage system participating in frequency modulation. The schematic diagram is shown in Figure 1.

In the serial control mode, the frequency modulation work distribution of the wind turbine and the ESS should be carried out according to the following principles,

$$
\begin{aligned}
\Delta P_{w_{\text {chu }}} & =\Delta P_{\text {vir-ine }}+\Delta P_{\text {change- } \omega} \\
\Delta P_{\text {vir-ine }} & =\frac{\frac{1}{2} m v^{2}}{\Delta t_{1}} \\
\Delta P_{\text {change } \beta} & =\frac{1}{2} \rho \pi R_{1}^{2} V_{m}^{3} C_{p} \\
C_{p} & =C_{p}(\beta+\Delta \beta) \\
\Delta P_{e_{\text {chu }}} & =\Delta P_{w+e}-\Delta P_{w_{\text {chu }}}
\end{aligned}
$$

where $m$ is the quality of wind turbine, $\mathrm{kg} ; \mathrm{v}$ is the speed of wind turbine, $\mathrm{m} / \mathrm{s} ; \Delta t_{1}$ is the response time for virtual inertia, $\mathrm{s} ; \rho$ is the air density, the standard value is $1.29 \mathrm{~kg} / \mathrm{m}^{3} ; R_{1}$ is the radius of sweep area, $\mathrm{m} ; V_{m}$ is wind speed, $\mathrm{m} / \mathrm{s} ; C_{p}$ is the percentage of wind energy utilization; $\beta$ is windward pitch angle of wind turbine; $\Delta \beta$ is the change of upwind pitch angle of wind turbine under serial control; $\Delta P_{w+e}$ Is the overall frequency modulation task volume of the wind storage system, MW; $\Delta P_{\text {vir-ine }}$ is the virtual inertial power regulation for wind turbines, $\mathrm{MW} ; \Delta P_{\text {change- } \omega}$ is the wind turbine pitch control to regulate the active power, MW; $\Delta P_{e_{c h u}}$ and $\Delta P_{w_{c h u}}$ are the adjustment of the ESS and the wind turbine, MW.

The frequency modulation flow chart using serial control is shown in Figure 2.

When the wind storage combined system responds to the system frequency modulation instruction by serial control mode, the wind turbine first responds to the frequency modulation instruction of the power system. At this time, the wind turbine controls its output power through virtual inertia and pitch control to suppress the frequency modulation of the power system. The unfinished frequency modulation components of wind turbines are allocated to the ESS.

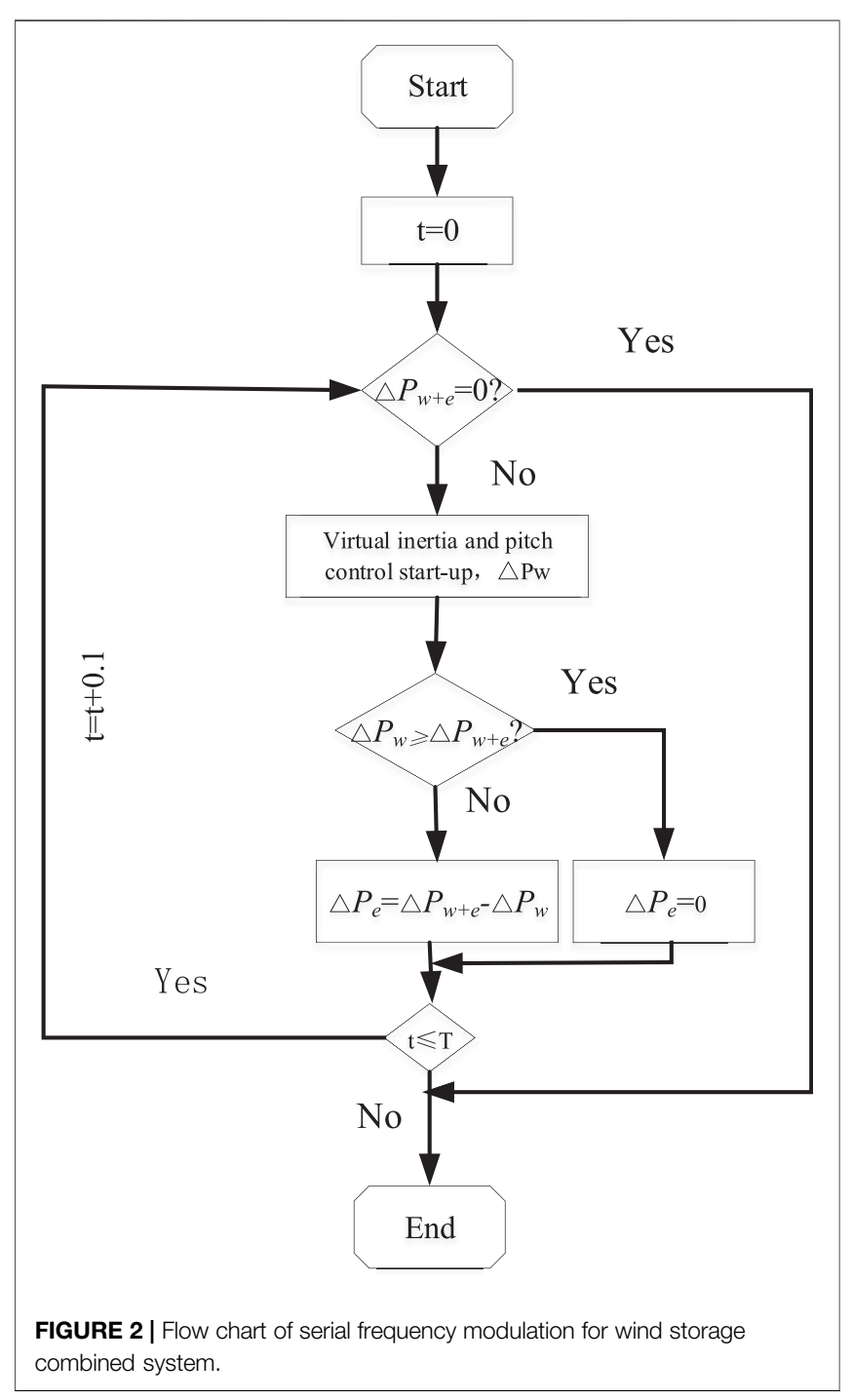




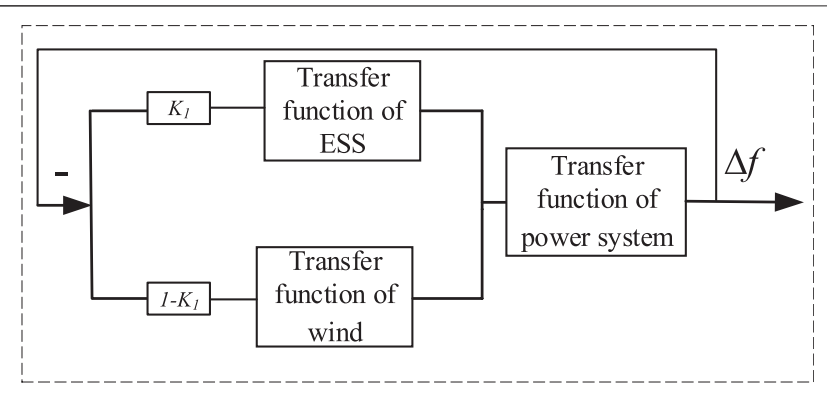

FIGURE 3 | Parallel frequency regulation schematic diagram of wind storage combined system.

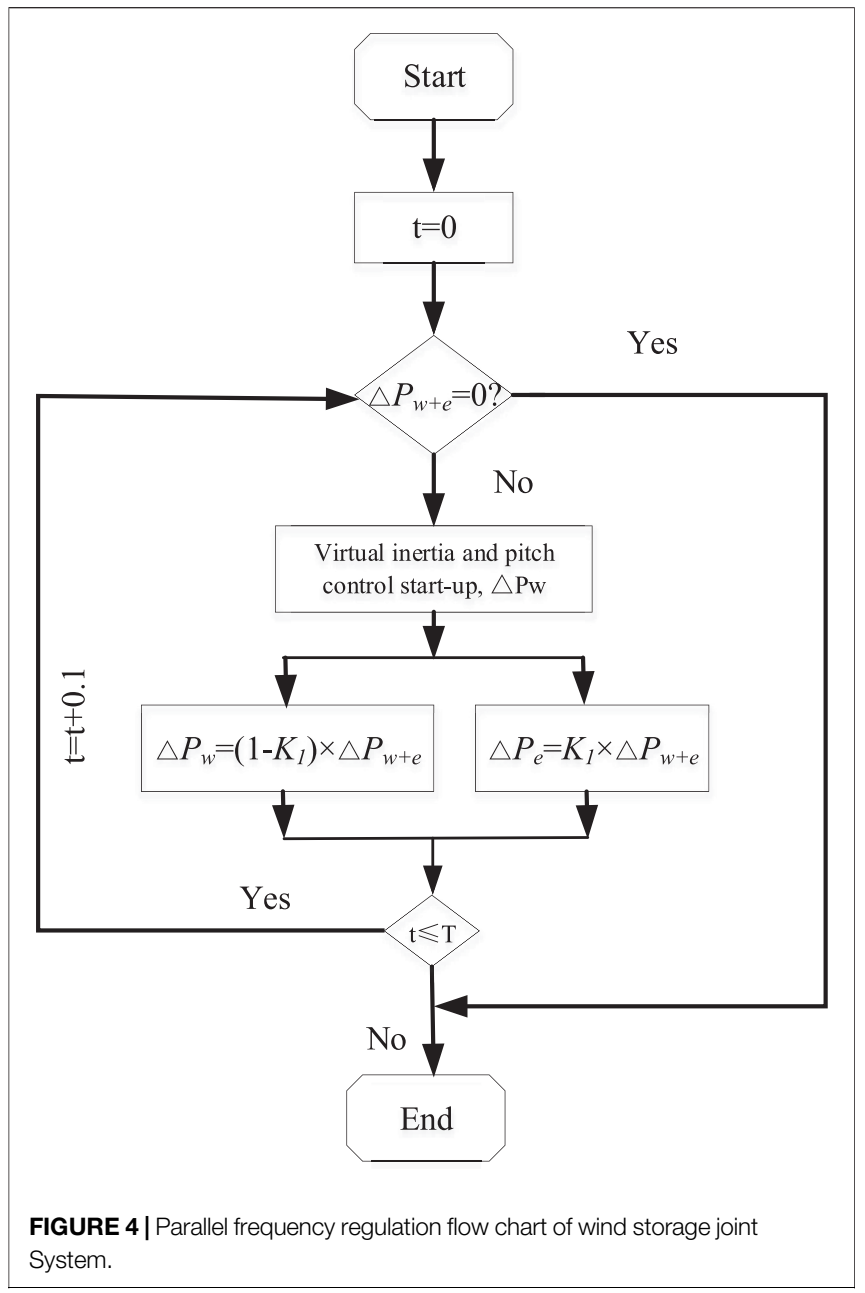

\section{Parallel Control Mode of Wind-Storage System}

When a parallel control strategy is adopted to participate in primary frequency modulation of power system, the control strategy sketch is shown in Figure 3.

Where $K_{1}$ is the control factor that the wind-storage system adopts a parallel control strategy to allocate tasks when receiving a frequency modulation instruction.

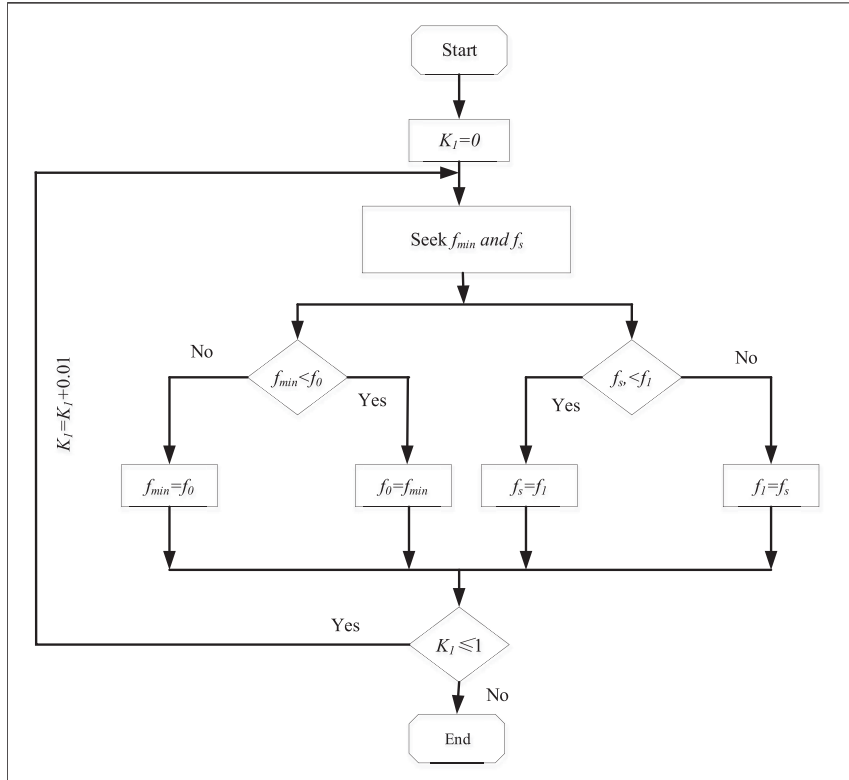

FIGURE 5 | Flow chart of parallel frequency regulation parameters calculation for wind storage combined system.

In the parallel control mode, the frequency modulation work distribution of wind turbine and ESS should be carried out according to the following principles,

$$
\begin{gathered}
\Delta P_{e_{\text {bin }}}=K_{1} * \Delta P_{w+e} \\
\Delta P_{w_{\text {bin }}}=\left(1-K_{1}\right) * \Delta P_{w+e} \\
\Delta P_{w_{\text {bin }}}=\Delta P_{\text {vir-ine }}^{\prime}+\Delta P_{\text {change- } \omega}^{\prime} \\
\Delta P_{\text {vir-ine }}^{\prime}=\frac{\frac{1}{2} m v_{1}^{2}}{\Delta t_{2}} \\
\Delta P_{\text {change- } \beta}^{\prime}=\frac{1}{2} \rho \pi R_{1}^{22} V_{m}^{3} C_{p}^{\prime}
\end{gathered}
$$

When adopting parallel control strategy, $\Delta P_{e_{b i n}}$ is the energy output of the ESS, MW; $v_{1}$ is the speed of wind turbine, $\mathrm{m} / \mathrm{s} ; \Delta t_{2}$ is the virtual inertial response time of wind turbines, $s ; \beta_{1} 、 \Delta \beta_{1}$ are the changes of pitch angle and pitch angle of wind turbine; $\Delta P_{\text {change- } \omega}^{\prime}, \Delta P_{\text {vir-ine }}^{\prime}$ are wind turbine pitch control and virtual inertia output, MW.

The schematic diagram of the process of wind storage system adopting parallel control strategy to participate in grid frequency modulation is shown in Figure 4.

When wind turbine adopts parallel control, the flow chart of $K_{1}$ value determination is shown in Figure 5.

Considering the actual operation requirements of the power system, the $K_{1}$ value in this paper is taken as 0.5 .

When adopting a parallel control strategy, the wind turbine and the ESS will respond to the primary frequency regulation commands of the power system according to their respective frequency modulation task coefficients $K_{1}$. Wind turbines change their output through virtual inertia and pitch control. The ESS will respond to frequency modulation instructions quickly within rated power. Wind turbines 


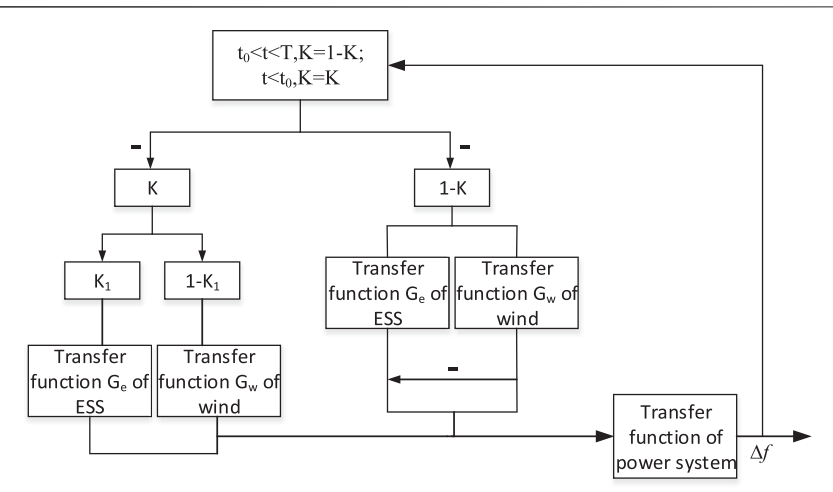

FIGURE 6 | Schematic diagram of optimal control strategy for wind storage system.

and ESS s work together to complete the primary frequency modulation task of the power system.

\section{Optimal Control Mode of Wind-Storage System}

The optimized control strategy proposed in this paper mainly considers the capacity allocation of the ESS and satisfies the constraints of serial control and parallel control. At each stage of wind power generation participating in power system frequency regulation, the control method is changed according to the actual situation and the operation status of the ESS to meet the power system frequency regulation needs.

\section{1) Target function construction}

Taking into account the actual operation requirements of the power system, the technical indicators of primary frequency modulation mainly include the maximum frequency deviation and the steady-state frequency deviation of the power system, and the economic indicators mainly include the capacity demand and power demand of the ESS. The objective function of the optimized control strategy of the wind storage system is constructed.

$$
\min \left[C_{1} \times\left(\Delta f_{d e v}+\Delta f_{s t a}\right)+C_{3} \times S\right]
$$

where $C_{1}$ and $C_{3}$ are the frequency modulation performance index penalty factor and energy storage cost penalty factor, both $0.5 ; \Delta f_{d e v}$ and $\Delta f_{\text {sta }}$ are the maximum frequency deviation and steady-state frequency deviation of the power system, respectively, HZ. $S$ is the cost coefficient that changes according to different control strategies.

The frequency modulation flow chart of the optimized control strategy is shown in Figure 6. Where $K$ and $1-K$ are the frequency allocation ratio of the ESS using serial control and parallel control, respectively; The system changes the proportion of series control and parallel control at time $t_{0}$.

When the wind storage system adopts the optimal control strategy, the total energy output of the wind storage system is as follows.

$$
\Delta P_{\text {you }}=\Delta P_{t<t_{0}}+\Delta P_{t_{0}<t \leq T}
$$

where $\Delta P_{t<t_{0}}$ and $\Delta P_{t_{0}<t \leq T}$ are the energy output of the windstorage combined system at time $0<\mathrm{t}<\mathrm{t}_{0}$ and time $\mathrm{t}_{0}<\mathrm{t}<\mathrm{T}$ under the optimized control strategy, respectively, MW; $\mathrm{T}$ is the maintenance time of primary frequency modulation, $30 \mathrm{~s}$.

$$
t \leq t_{0}\left\{\begin{array}{l}
\Delta P_{t<t_{0}}=\Delta P_{c h u 1}+\Delta P_{b i n 1} \\
\Delta P_{c h u 1}=\Delta P_{e c h u 1}+\Delta P_{w c h u 1} \\
\Delta P_{b i n 1}=\Delta P_{e b i n 1}+\Delta P_{w b i n 1} \\
\Delta P_{\text {echu } 1}=\Delta P_{w+e}(1-K)-\Delta P_{w c h u 1} \\
\Delta P_{w b i n 1}=\Delta P_{w+e} \times K \times\left(1-K_{1}\right) \\
\Delta P_{\text {ebin } 1}=\Delta P_{w+e} \times K \times K_{1} \\
\Delta P_{w c h u 1}=\Delta P_{\text {vir-ine } 1}+\Delta P_{\text {change- } \beta 1}
\end{array}\right.
$$

In the period of $0<\mathrm{t}<\mathrm{t}_{0}, \Delta P_{c h u 1}$ and $\Delta P_{b i n 1}$ are the total output of the serial control and parallel control of the wind storage system under the optimized control strategy, respectively, MW. $\Delta P_{w c h u 1}$ and $\Delta P_{w b i n}$ are the output of the serial control and parallel control of the wind turbine under the optimized control strategy, respectively, MW. $\Delta P_{e c h u 1}$ and $\Delta P_{e b i n 1}$ are the output of serial control and parallel control of ESS under optimized control strategy, respectively, MW.

$$
t_{0}<t \leq T\left\{\begin{array}{l}
\Delta P_{t_{0}<t \leq T}=\Delta P_{\text {chu } 2}+\Delta P_{\text {bin } 2} \\
\Delta P_{\text {chu } 2}=\Delta P_{\text {echu } 2}+\Delta P_{\text {whu } 2} \\
\Delta P_{\text {bin } 2}=\Delta P_{\text {ebin } 2}+\Delta P_{\text {win } 2} \\
\Delta P_{\text {echu } 2}=\Delta P_{w+e} \times K-\Delta P_{\text {whiu } 2} \\
\Delta P_{w b i n 2}=\Delta P_{w+e} \times(1-K) \times\left(1-K_{1}\right) \\
\Delta P_{\text {ebin } 2}=\Delta P_{w+e} \times(1-K) \times K_{1} \\
\Delta P_{\text {whhu } 2}=\Delta P_{\text {vir-ine } 2}+\Delta P_{\text {change- } \beta 2}
\end{array}\right.
$$

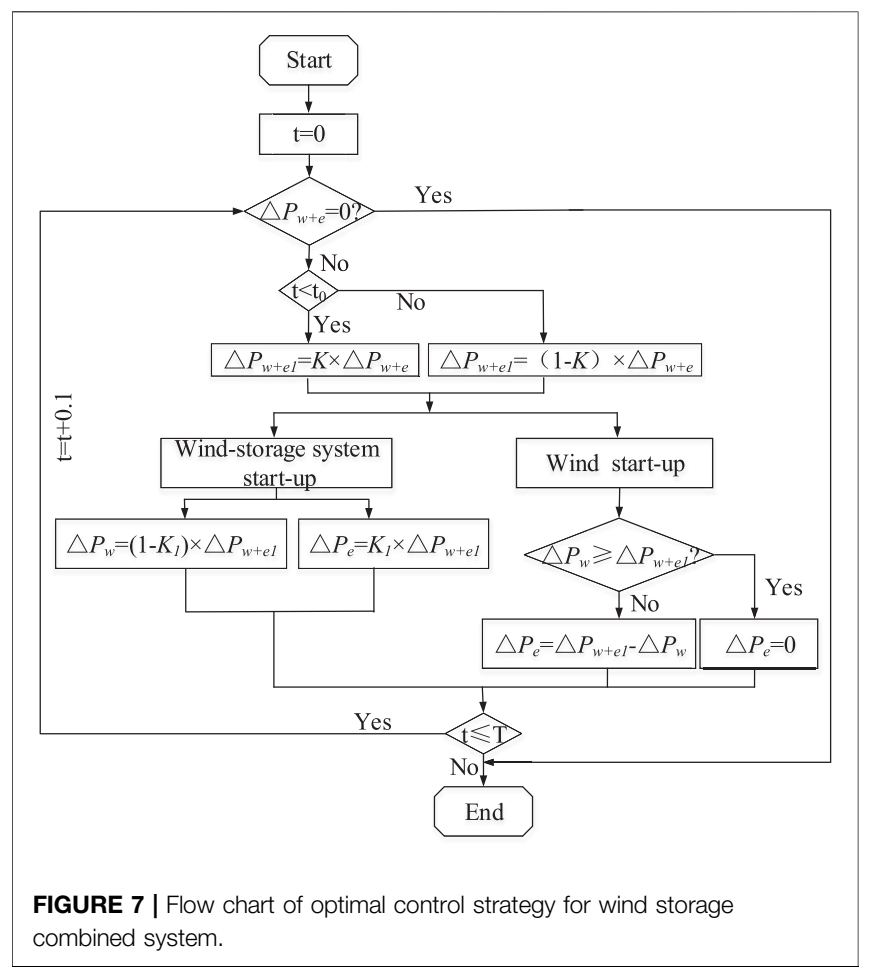



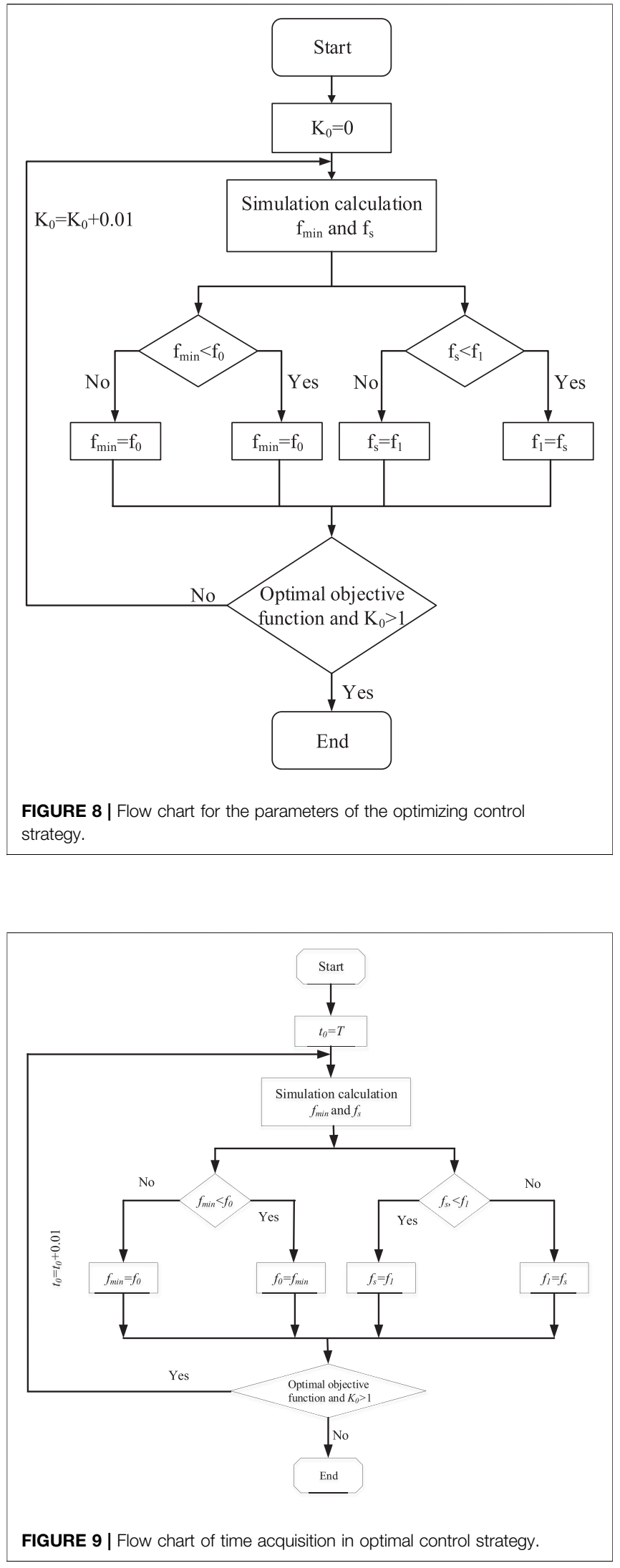

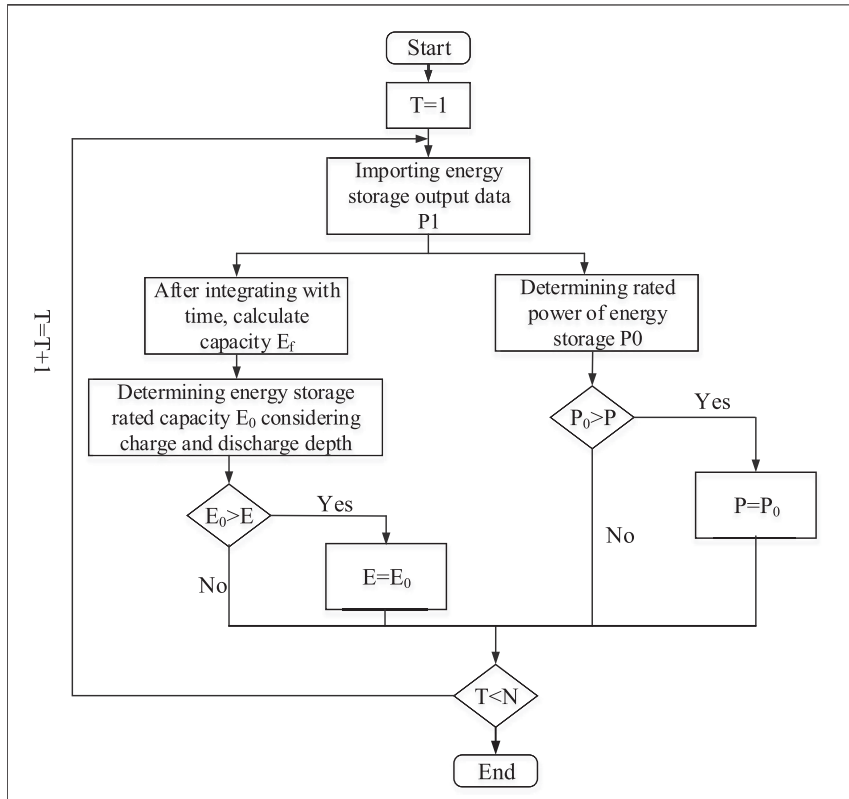

FIGURE 10 | Flow chart of capacity allocation for ESS.

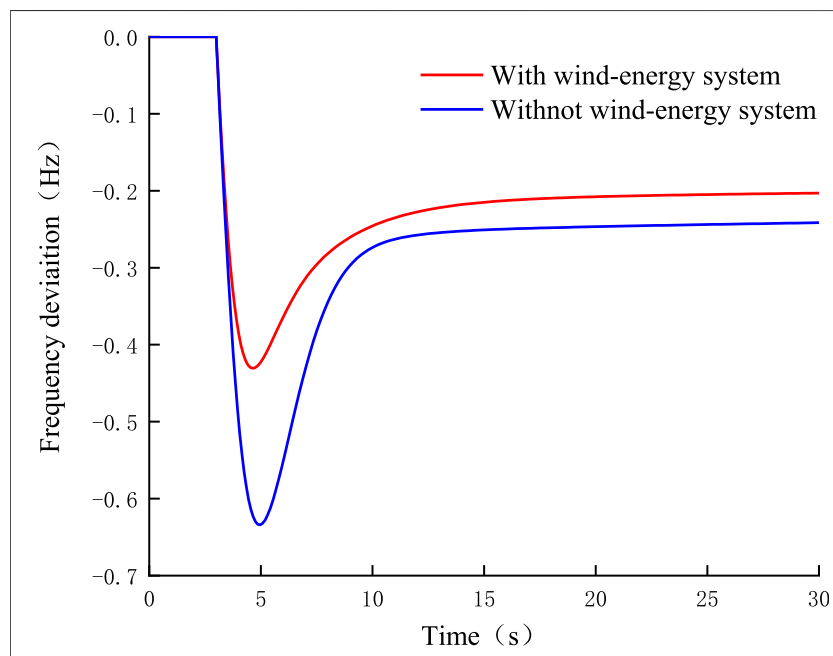

FIGURE 11 | Frequency regulation results with/without wind power and ESS.

In the formula, the number 2 indicates that the time period is $\mathrm{t}_{0}<\mathrm{t}<\mathrm{T}$.

The optimized control strategy operation flowchart is shown in Figure 7.

The purpose of the optimal control strategy is to reduce the frequency fluctuation of the power system, and reduce the cost of energy storage, and improve the economy. 


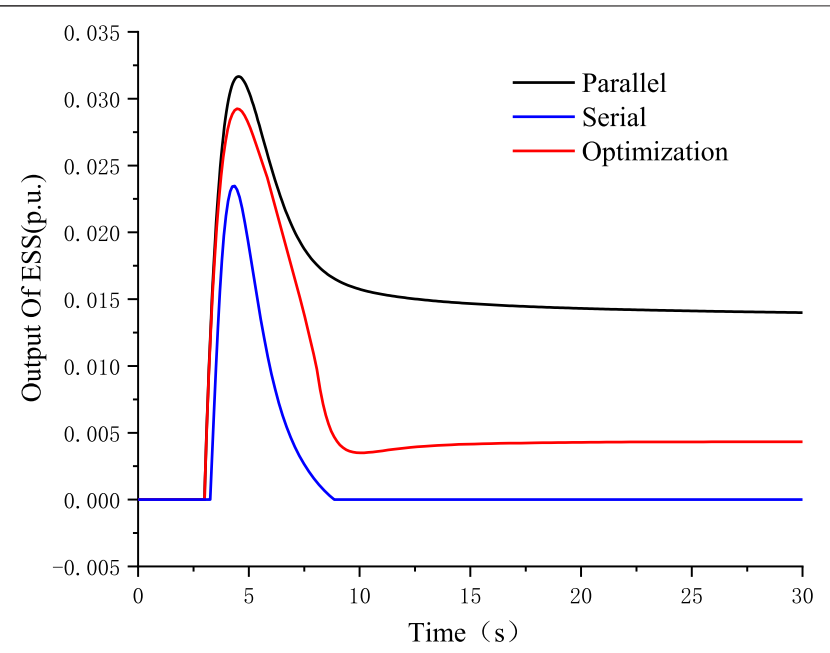

FIGURE 12 | Comparison of energy storage capacity under different control strategies.

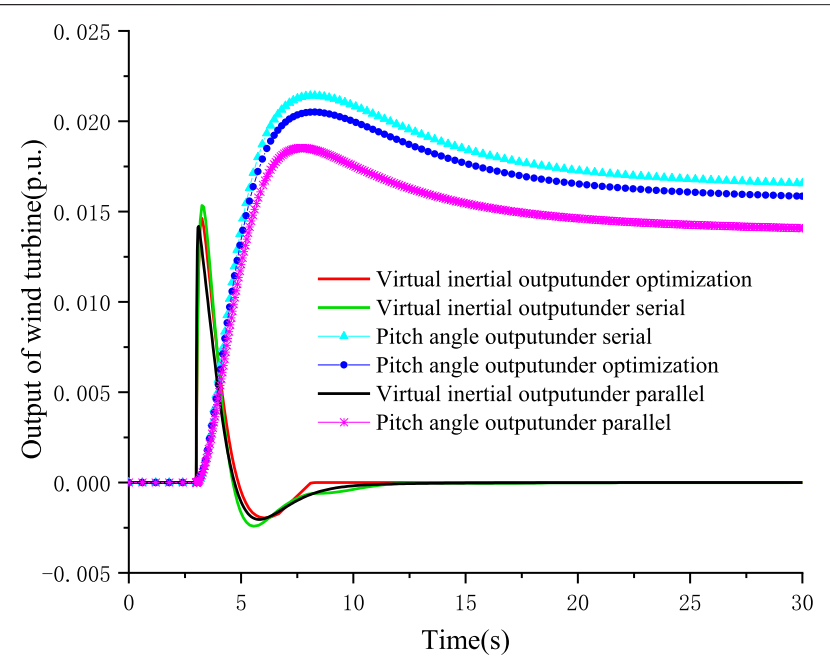

FIGURE 13 | Comparison of wind power under different control strategies.

2) Setting control parameters

Calculate $K$ and $t_{0}$ with the control variable method. The calculation method is shown in Figure 8. Where $K_{0}$ is the initial set value; $f_{\min }$ is the lowest frequency drop point in the simulation process; $f_{\mathrm{s}}$ is the steady-state frequency value. When the value of $K$ is 0.85 , the overall frequency modulation effect of the system is the best.

The calculation method of $t_{0}$ is shown in Figure 9. Where $T_{n}$ is the initial time set point. Through simulation verification, when $t_{0}$ takes $0.45 \mathrm{~s}$, the frequency modulation effect of the system is the best.

\section{Demand Analysis of ESS Capacity}

This section mainly solves the problem of capacity allocation of the ESSs, including the determination of the constraints of capacity allocation of the ESSs and the establishment of configuration processes.

\section{Constraints of ESS Configuration}

Energy storage systems need to meet the constraints of state of charge (SOC). At a certain time t, SOC is calculated by the following formula:

$$
S O C_{t}=S O C_{t-T}+\frac{\int_{t_{0}}^{t} P_{e} d t}{E}
$$

where $t_{1}$ is the time when the ESS starts to work, $s ; E$ is the ESS rated capacity, MWh.

The SOC should meet the constraints of the upper and lower limits:

$$
S O C_{\min } \leq S O C \leq S O C_{\max }
$$

$S O C_{\min }$ and $S O C_{\max }$ are the lower and upper limits of chargedischarge of the ESS, respectively, 0.05-0.95.

The ESS should also satisfy the output power $P_{e}$ constraint, as follows:

$$
P_{\min } \leq P_{e} \leq P_{\max }
$$

Under normal operation, $P_{\min }$ and $P_{\max }$ are the lower limit and upper limit of the output power of the energy storage system, respectively, MW.

\section{ESS Configuration Method}

The configuration method of the capacity and power of the ESS is as follows:

1) $\mathrm{N}=1$, import the first frequency data;

2) The output data $P_{1}$ of the ESS is derived and the rated power $P$ of the ESS is obtained;

3) The capacity $E_{1}$ of the ESS is calculated according to the energy output during the frequency modulation period;

4) Considering the charging and discharging efficiency of the ESS, the rated capacity E of the ESS can be obtained;

5) $\mathrm{N}=\mathrm{N}+1$.

In summary, the following Figure $\mathbf{1 0}$ configuration flowchart can be obtained.

The energy storage system configuration results of the three control strategies are shown in Table 1.

\section{EVALUATION METHOD OF FREQUENCY MODULATION EFFECT}

The frequency modulation effect evaluation method proposed in this paper comprehensively analyzes the frequency modulation effect and economy and can be used to compare the comprehensive frequency modulation performance of different control strategies.

\section{Evaluation System}

The calculation formula of comprehensive frequency modulation performance evaluation is as follows: 
TABLE 1 | Configuration results of three control strategies

\begin{tabular}{lcc}
\hline Control strategy & Rated power (MW) & Rated capacity (MWh) \\
\hline Parallel control & 6.54 & 1.062 \\
Serial control & 4.84 & 0.524 \\
Optimize control & 5.84 & 0.636 \\
\hline
\end{tabular}

$$
G=\left(\sum_{i=1}^{n} g_{i} * D_{i}\right) / n
$$

$G$ is the score of the final frequency modulation effect; $g_{i}$ is the score of the $i$-th factor affecting the effect of frequency modulation; $D_{i}$ is the weighting factor of the $i$-th factor that affects the frequency modulation effect; $n$ is the number of factors that affect the frequency modulation effect.

The calculation method of the maximum frequency deviation $G_{d e v}$ of the power grid is as follows:

$$
G_{d e v}=\frac{\Delta f_{d e v}-f_{d e v-x i a}}{f_{d e v-s h a}-f_{d e v-x i a}} *\left(G_{d e v-s h a}-G_{d e v-x i a}\right)+G_{d e v-x i a}
$$

$f_{d e v-x i a}$ and $f_{d e v-s h a}$ are the lower limit frequency and upper limit frequency of the frequency change range $\Delta f_{d e v}$, respectively; $G_{d e v-x i a}$ and $G_{d e v-s h a}$ are the lower limit score and upper limit score in the frequency change range $\Delta f_{d e v}$, respectively.

The calculation method of power system steady-state frequency deviation $G_{s t a}$ is as follows:

$$
G_{s t a}=\frac{\Delta f_{\text {sta }}-f_{\text {sta-xia }}}{f_{\text {sta-sha }}-f_{\text {sta-xia }}} *\left(G_{\text {sta-sha }}-G_{\text {sta-xia }}\right)+G_{\text {sta-xia }}
$$

where $f_{\text {sta-xia }}$ and $f_{\text {sta-sha }}$ are the lower limit frequency and upper limit frequency of the frequency change range $\Delta f_{\text {sta }}$, respectively; $G_{\text {sta-xia }}$ and $G_{\text {sta-sha }}$ are the lower limit score and upper limit score in the frequency change range $\Delta f_{\text {sta }}$, respectively.

The calculation formula of the power system frequency modulation economy $G_{e c o}$ score is as follows:

$$
G_{e c o}=\frac{P_{f r e-c b}}{P_{f r e-s y}} *\left(G_{e c o-s h a}-G_{e c o-x i a}\right)+G_{e c o-x i a}
$$

where $P_{f r e-c b}$ and $P_{f r e-s y}$ are the cost and revenue of energy storage system participating in frequency modulation, respectively; $G_{e c o-x i a}$ and $G_{e c o-s h a}$ are the lower limit and upper limit of the score in the range, respectively.

\section{Evaluation Criterion}

Table 2 gives the scoring principles for evaluating the comprehensive performance of frequency modulation:

Considering that the limit value of power system underfrequency load shedding is $0.5 \mathrm{~Hz}$, the lowest frequency limit in the table is set to $0.5 \mathrm{~Hz}$; In the case of limit wind power permeability (0.5), the steady frequency of primary frequency modulation is $0.6 \mathrm{~Hz}$ under the same disturbance, so $0.6 \mathrm{~Hz}$ is set as the upper limit of steady frequency value. Because there is no compensation in the primary frequency modulation market at present in China when the total benefit-cost ratio of primary frequency modulation in power system is greater than or equal to 1 , the score is the highest, and vice versa, the score decreases.

\section{SIMULATION ANALYSIS}

\section{Simulation Parameters}

The method described in this paper is based on the windows 10 64 bit operating system, and the relevant programs are written using matlab 2018b software, Visio2013 is used for flowchart drawing, and Origin2018 software is used for waveform drawing. with the load set to $1000 \mathrm{MW}$, the wind farm rated power of $200 \mathrm{MW}$, and the load fluctuation of $100 \mathrm{MW}$ (0.1p.u).

\section{Simulation Results With Different Control Strategies}

First of all, it is necessary to verify whether the configuration of the wind-storage system has a positive effect on power system frequency modulation。 When adopting the optimal control strategy, perform the following Figure 11 simulation analysis:

The results prove that the configuration of wind/storage system is conducive to power system frequency modulation. Table 3 shows the specific values of the impact of the configuration of the wind storage system on frequency, including steady-state frequency deviation and maximum frequency deviation.

With the addition of wind storage system, the lowest frequency is increased by $0.22 \mathrm{~Hz}$ and the steady frequency is increased by $0.044 \mathrm{~Hz}$.

\section{Contribution of Frequency Modulation Resources Under Different Control Strategies}

The following is an analysis of two types of frequency modulation resources, including energy storage systems and wind farm.

\section{1) Energy storage system output}

The primary frequency modulation output of the energy storage system under the same disturbance is simulated using parallel, serial and optimal control strategies. The simulation results are shown in the following Figure 12.

In the process of traditional frequency modulation resource startup, the energy storage system responds quickly to frequency changes after disturbances occur in the power system. The energy storage output rises rapidly from 0 to the maximum in $0.3 \mathrm{~s}$. The maximum energy storage output is 0.03 p.u under parallel control, 0.023 p.u under serial control and 0.03 p.u under optimal control. Therefore, parallel control and optimal control of the energy storage system can provide greater energy support at the early stage of disturbance occurrence and raise the frequency to the lowest point.

The use of energy storage resources in parallel control is stable at 0.015 p.u., the series control is almost 0 , and the optimal control is between the two, stable at 0.0047 p.u. The optimized control of the 
TABLE 2 | Score table of frequency regulation effect.

\begin{tabular}{|c|c|c|c|c|c|c|}
\hline Maximum deviation & $\leq 0.2$ & $0.2 \sim 0.3$ & $0.3 \sim 0.4$ & $0.4 \sim 0.5$ & \multicolumn{2}{|c|}{$\geq 0.5$} \\
\hline Score & 10 & $8 \sim 10$ & $6 \sim 8$ & $4 \sim 6$ & \multicolumn{2}{|c|}{$\leq 3$} \\
\hline Steady deviation & $\leq 0.2$ & $0.2 \sim 0.25$ & $0.25 \sim 0.3$ & $0.3 \sim 0.35$ & $0.35 \sim 0.4$ & 0.4 \\
\hline Score & 10 & $8 \sim 10$ & 6 8 & $4 \sim 6$ & $2 \sim 4$ & $<2$ \\
\hline Cost & $\leq \mathrm{P}$ & $\mathrm{P} \sim 1.2 \mathrm{P}$ & 1.2P 1.4P & 1.4P 1.6P & 1.6P 1.8P & $\geq 1.8 \mathrm{P}$ \\
\hline Score & 10 & $8 \sim 10$ & $6 \sim 8$ & $4 \sim 6$ & $2 \sim 4$ & $\leq 2$ \\
\hline
\end{tabular}

TABLE 3 | Frequency deviation with/without wind power and ESS.

Data type

With wind-storage system

Without wind-storage system
Maximum deviation (Hz)

$-0.425$

$-0.647$
Steady deviation $(\mathrm{Hz})$

$-0.205$

$-0.249$
TABLE 4 | Frequency deviation comparison.

\begin{tabular}{lcc} 
Control strategy & Maximum deviation $\mathbf{( H z )}$ & Steady deviation $(\mathbf{H z})$ \\
\hline Parallel control & -0.387 & -0.187 \\
Serial control & -0.442 & -0.241 \\
Optimize control & -0.401 & -0.205
\end{tabular}

energy storage output power is small, that is, the use of energy storage resources is small, and it can provide backup capacity for the next frequency modulation work.

2) Wind farm output

Wind farms are an important part of the frequency modulation resources of power systems. The control strategy should consider improving the frequency modulation performance of wind farms. The following Figure $\mathbf{1 3}$ is an analysis of the wind farm frequency modulation capabilities of three different control strategies.

In the start-up stage of wind turbine, the virtual inertia response of wind farm under each control strategy releases the kinetic energy of wind turbine rotor within $0.1 \mathrm{~s}$. This will delay the fast frequency drop of power system, and each control strategy has little influence on this process.

In the process of pitch control, because wind power takes on different tasks in different control strategies, the control effect of the control strategy is reflected in the process of pitch output. Serial control mainly uses wind power as the backup energy for ESS, so the maximum output power of wind power is $0.024 \mathrm{p}$. u. In parallel control, wind power and energy storage work in parallel mode, so the output power of wind power is smaller than 0.017 p. u., and the optimal control strategy is 0.021 p.u. between the above two. This proves that the optimized control strategy can reasonably use the capacity of wind power frequency modulation and reduce the amount of energy storage action.

\section{Frequency Modulation Effect Comparison}

The following Supplementary Figure S1 is a simulation analysis of the frequency modulation effects of the three control strategies using Simulink.

Table 4 is the frequency modulation effect data of three control strategies, including maximum frequency deviation and steady-state frequency deviation.

It can be drawn from the table above that the maximum frequency deviation and the steady-state frequency deviation of the optimized control strategy are both between serial control and parallel control, and are superior to serial control.

Generally speaking, the optimal control strategy can provide effective support for the power system by utilizing the fast response characteristics of the energy storage system in the start-up stage of traditional units and wind power. After the start-up of the unit, on the premise of maximizing the frequency modulation capability of the traditional unit, the energy storage capacity is reduced and the economy of frequency modulation is guaranteed.

\section{Cost Analysis Under Different Control Strategies}

Taking the lithium batteries which are widely used in frequency modulation of power system as the research object, the economy of lithium batteries under three control strategies is analyzed. The simulation parameters are shown in the following Table 5.

There are no compensation measures for battery energy storage participating in primary frequency modulation of power grid (Chen et al., 2016). But battery energy storage can be recycled. For example, lithium batteries can recycle non-ferrous metals for secondary use

TABLE 5 | Economy parameters of frequency regulation.

\begin{tabular}{lcccc}
\hline Parameter name & Recovery cost & $\begin{array}{c}\text { Abandoned wind } \\
\text { income }\end{array}$ & $\begin{array}{c}\text { Unit power } \\
\text { price }\end{array}$ & $\begin{array}{c}\text { Unit capacity } \\
\text { price }\end{array}$ \\
\hline Parameter values & $4,046 \$ / t$ & $80 \$ / \mathrm{MWh}$ & $738,462 \$ / \mathrm{MW}$ & $215,385 \$ / \mathrm{MWh}$ \\
\hline
\end{tabular}


TABLE 6 | Cost comparison under different control strategies.

\begin{tabular}{lcc}
\hline Control strategy & Profit (\$) & Cost (\$) \\
\hline Parallel Control & $1,428,154$ & $5,058,277$ \\
Serial Control & $1,026,262$ & $3,687,015$ \\
Optimize Control & $1,177,108$ & $4,449,600$
\end{tabular}

of frequency modulation in the power grid. Considering the effect of frequency modulation under different control strategies and the economic factors of wind storage combined system participating in primary frequency modulation, a comprehensive evaluation method of primary frequency modulation are designed.

TABLE 7 | Scoring table for different control strategies.

\begin{tabular}{|c|c|c|c|c|}
\hline Control strategy & Lowest frequency score & Frequency steady score & Cost score & Total score \\
\hline Parallel control & 7.2 & 10 & 4.255 & 6.427 \\
\hline Serial control & 5.95 & 9.35 & 5.59 & 6.72 \\
\hline Optimize control & 7.05 & 9.75 & 5.92 & 7.122 \\
\hline
\end{tabular}

(Li et al). At the same time, the use of energy storage systems can also reduce wind curtailment. Table 6 compares the costs and profits of energy storage systems.

From the above data, it can be concluded that the cost of the parallel control strategy under the same interference is the highest. Optimal control reduces the energy storage workload after the traditional frequency modulation resources are started, so the economy is better. Serial control has lower revenue due to less initial power and capacity.

According to the evaluation method proposed in Chapter 4, the three control strategies are scored and compared. The scores are shown in Table 7.

According to the results in the above table, the overall score of optimized control is higher than that of serial control and parallel control, which proves that the overall performance of optimized control is higher than that of serial control and parallel control after a comprehensive analysis of the economy and frequency modulation effect.

\section{CONCLUSION}

Aiming at the control strategy of large-scale wind storage system participating in power system primary frequency modulation, this paper made the following work:

In this paper, the serial and parallel control modes of ESS and wind turbine are studied based on the traditional frequency modulation mode of power system. Considering the economy and frequency modulation effect, an optimal control strategy based on serial and parallel of wind-storage system is proposed, and the objective function of the optimal control strategy is established. And the control variable method is used to optimize the relevant variables in the optimization strategy.

The comprehensive evaluation method of wind storage combined system participating in primary frequency regulation of power grid is studied. Considering that the current primary frequency modulation evaluation method cannot meet the requirement of paying equal attention to both the effect of frequency modulation and the economy
In the analysis part of the calculation example, the same interference is added to the three different control strategies, and the frequency modulation effect is analyzed using the simulation system. The results show that the optimized control reduces the maximum frequency deviation by $0.041 \mathrm{~Hz}$ and the steady-state frequency deviation by $0.036 \mathrm{~Hz}$ than the serial control. Compared with the parallel control strategy, the rated power is reduced by $0.7 \mathrm{MW}$ and the rated capacity is reduced by $0.462 \mathrm{MWh}$.

According to the proposed evaluation method, the comprehensive frequency modulation effect scores of the three control strategies are evaluated. The results show that the optimal control strategy proposed in this paper scores 7.122 points, which is higher than 6.72 points for serial control and 6.427 points for parallel control. This proves that the overall performance of optimized control is higher than that of serial control and parallel control after a comprehensive analysis of the economy and frequency modulation effect.

\section{OUTLOOK}

This paper optimizes the parallel and serial control in the traditional frequency modulation method of wind storage system, and puts forward the optimal control strategy of this paper, and finally achieved certain results. At the same time, this paper still has some areas that can be improved. For example, the effect of the optimal control strategy proposed in this paper may not be better than the serial control strategy and the parallel control strategy under certain conditions. The optimal control strategy is the optimal method proposed after comprehensively considering the cost of energy storage and the effect of frequency modulation. It may not be applicable in some occasions where the accuracy of frequency modulation needs to be improved (while the cost of frequency modulation is allowed to be ignored). In fact, the cost of the current energy storage system is still high, so its economy is still a very important indicator in the power system. If the cost of the energy storage system can be significantly reduced in the 
future, then this strategy still has room for continuous improvement.

\section{DATA AVAILABILITY STATEMENT}

The original contributions presented in the study are included in the article/Supplementary Material, further inquiries can be directed to the corresponding author.

\section{AUTHOR CONTRIBUTIONS}

CL: Conceptualization, Methodology, Writing- Original draft preparation. ZZ: Writing- Original draft preparation. JL: Methodology. YM: Data curation. JZ: Translation and Validation.

\section{REFERENCES}

Annamraju, A., and Nandiraju, S. (2019). Robust Frequency Control in a Renewable Penetrated Power System: an Adaptive Fractional Order-Fuzzy Approach. Prot. Control. Mod. Power Syst. 4 (4), 181-195. doi:10.1186/ s41601-019-0130-8

Ayyarao, T. S. L. V., and Ayyarao, V. (2019). Modified Vector Controlled DFIG Wind Energy System Based on Barrier Function Adaptive Sliding Mode Control. Prot. Control. Mod. Power Syst. 4 (4), 34-41. doi:10.1186/s41601019-0119-3

Chen, Dayu., Zhang, Lizi., and Wang, Liguo. (2016). Control Strategy of Energy System for Frequency Regulation and Evaluation of Investment Income. Mod. Electric Power 33 (1), 80-86. doi:10.19725/j.cnki.1007-2322.2016.01.013

Dang, J., Seuss, J., Suneja, L., and Harley, R. G. (2014). SoC Feedback Control for Wind and ESS Hybrid Power System Frequency Regulation. IEEE J. Emerg. Sel. Top. Power Electron. 2 (1), 79-86. doi:10.1109/jestpe.2013.2289991

$\mathrm{Hu}$, Zechun., Xia, Rui., and $\mathrm{Wu}$, Linlin. (2016). Joint Operation Optimization of Wind-Storage Union with Energy Storage Participating Frequency Regulation. Power Syst. Tech. 40 (08), 2251-2257. doi:10.13335/j.1000-3673.pst.2016.08.001

$\mathrm{Hu}$, Zechun., Xu, X. I. E., and Zhang, Fang. (2014). Research on Automatic Generation Control Strategy Incorporating Energy Storage Resources. Proc. CSEE 34 (29), 5080-5087. doi:10.13334/j.0258-8013.pcsee.2014.29.009

Jing, L. I., Fang, Yong., and Song, Jiahua. (2005). Research on Subsection and Layer Control Strategy of Doublyfed Variable Speed Wind Turbine. Power Syst. Tech. 29 (9), 15-21. doi:10.13335/j.1000-3673.pst.2005.09.004

Kundur, Prabha. (2001). Power System Stability and Control. Beijing: China Electric Power Press.

Li, J., Fu, Y., Li, C., Li, J., Xing, Z., and Ma, T. (2021). Improving Wind Power Integration by Regenerative Electric Boiler and Battery Energy Storage Device. Int. J. Electr. Power Energ. Syst. 131 (131), 107039. doi:10.1016/ j.ijepes.2021.107039

Li, Jianbo., Zheng, X. U., and Ji, Zhongguang. An Overview on the Current Technologies of Recycling Spent Lithium-Ion Bat-Teries. Chin. J. Rare Met. 43 (02), 201-212. doi:10.13373/j.cnki.cjrm.xy17060021

Li, Jianlin., Ma, Huimeng., and Yuan, Xiaodong. (2017). Overview on Key Applied Technologies of Large-Scale Distributed Energy Storage. Power Syst. Tech. 41 (10), 3365-3375. doi:10.13335/j.1000-3673.pst.2017.1101

Li, Junhui., Feng, Xichao., Yan, Gangui., Ge, Y., Li, D., and Fu, Y. (2018). Survey on Frequency Regulation Technology in High Wind Penetration Power System. Power Syst. Prot. Control. 46, 163-170. doi:10.7667/PSPC170002

Luo, F., Meng, K., Dong, Z. Y., Zheng, Y., Chen, Y., and Wong, K. P. (2015). Coordinated Operational Planning for Wind Farm with Battery Energy Storage System. IEEE Trans. Sustain. Energ. 6 (1), 253-262. doi:10.1109/ tste.2014.2367550

Miao, Fufeng., Tang, Xisheng., and Qi, Zhiping. (2015). Analysis of Frequency Characteristics of Power System Based on Wind Farm-Energy Storage Combined Frequency Regulation. High Voltage Eng. 41 (07), 2209-2216.

\section{FUNDING}

This work was supported in part by Joint Foundation of Natural Science Foundation of Jilin Province (No. 2020122352JC) and the National Natural Science Foundation of China (No. U1766204).

\section{SUPPLEMENTARY MATERIAL}

The Supplementary Material for this article can be found online at: https://www.frontiersin.org/articles/10.3389/fenrg.2021.739439/ full\#supplementary-material

SUPPLEMENTARY FIGURE S1 | Comparison chart of frequency modulation effect.

Muyeen, S. M., Hasanien, H. M., and Tamura, J. (2012). Reduction of Frequency Fluctuation for Wind Farm Connected Power Systems by an Adaptive Artificial Neural Network Controlled Energy Capacitor System. IET Renew. Power Gener. 6 (4), 226. doi:10.1049/iet-rpg.2010.0126

Pandzic, H., and Bobanac, V. (2019). An Accurate Charging Model of Battery Energy Storage. IEEE Trans. Power Syst. 34 (2), 1416-1426. doi:10.1109/ TPWRS.2018.2876466

Peng, L. I., Dou, Pengchong., Li, Yuwei., and Fengquan, Z. (2015). Application of Microgrid Technology in Active Distribution Network. Electric Power Automation Equipment 35 (4), 8-16. doi:10.16081/j.issn.1006-6047.2015.04.002

Ramtharan, G., Jenkins, N., and Ekanayake, J. B. (2007). Frequency Support from Doubly Fed Induction Generator Wind Turbines. IET Renew. Power Gener. 1 (1), 3-0. doi:10.1049/iet-rpg:20060019

Shen, Guanye., Chen, L. I., and Xu, Bingliang. (2018). Economic Allocation for Energy Storage System Considering Wind Power. J. Northeast Electric Power Univ. 38 (04), 27-34.

Sun, Bohao., Tang, Yong., and Lin, Y. E. (2019). Integrated Frequency Control Strategy for Wind Power Cluster with Mul-Tiple Temporal-Spatial Scale Coordination Based on H-DMPC. Proc. CSEE 39 (1), 155-167. doi:10.1109/ tec.2015.2476827

Sun, Yi., Li, Zekun., and Liu, Di. (2018). An Optimization Control Strategy for Intelligent UFLS Using Dynamically Aggregated Controllable Loads. Proc. CSEE 38 (7), 1913-1921+2207. doi:10.13334/j.0258-8013.pcsee.1707407

Tan, Y., Meegahapola, L., and Muttaqi, K. M. (2016). A Suboptimal Power-PointTracking-Based Primary Frequency Response Strategy for DFIGs in Hybrid Remote Area Power Supply Systems. IEEE Trans. Energ. Convers. 31 (1), 93-105. doi:10.1109/tec.2015.2476827

Tariq, M., Maswood, A. I., Gajanayake, C. J., and Gupta, A. K. (2018). Modeling and Integration of a Lithium-Ion Battery Energy Storage System with the More Electric Aircraft 270 V DC Power Distribution Architecture. IEEE Access 6, 41785-41802. doi:10.1109/ ACCESS.2018.2860679

Teninge, A., Jecu, C., Roye, D., Bacha, S., Duval, J., and Belhomme, R. (2009). Contribution to Frequency Control through Wind Turbine Inertial Energy Storage. IET Renew. Power Gener. 3 (3), 358 doi:10.1049/ietrpg.2008.0078

Wind power grid-connected operation in the first three quarters (2019). Wind Power Grid-Connected Operation in the First Three Quarters of 2019. Beijing: National Energy Administration.

Wu, Jun., Lu, Zongxiang., Qiao, Ying., et al. (2018). Optimal Operation of Wind Farm with Hybrid Storage Devices Considering Efficiency Characteristics of Dynamic Charging and Discharging. Automation Electric Power Syst. 42 (11), 41-47. doi:10.7500/AEPS20180326002

Wu, Zishuang., Yu, Jilai., and Peng, Xiyun. (2013). DFIG's Frequency Regulation Method Only for High Wind Speed with Subop-Timal Power Tracking. Trans. China Electrotechnical Soc. 28 (05), 112-119.

Xing, Pengxiang., Shi, Qiaoming., Wang, Gang., Fu, L., Wang, Y., and Wu, Y. (2018). Response Characteristics and Mechanism Analysis about Virtual Inertia 
Control of Wind Generators. High Voltage Eng. 44 (04), 1302-1310. doi:10.13336/j.1003-6520.hve.20180329033

Yan, Gangui., Wang, Yibo., and Cheng, Z. H. O. N. G. (2016). Frequency Control Strategy for Wind Storage Combined System. Electric Power Construction 37 (12), 55-60. doi:10.3969/j.issn.1000-7229.2016.12.007

Yan, Gangui., and Zhao, Weizhe. (2018). ZHANG Liyu Research on Integrated Control of Deloading Frequency Regulation for Variable Speed and Variable Pitch Angle Wind Turbines. J. Northeast Electric Power Univ. 38 (05), 1-8.

Zertek, A., Verbic, G., and Pantos, M. (2012). A Novel Strategy for Variable-Speed Wind Turbines' Participation in Primary Frequency Control. IEEE Trans. Sustain. Energ. 3 (4), 791-799. doi:10.1109/tste.2012.2199773

Zhang, Xu., Chen, Yunlong., Yue, Shuai., Zha, X., and Zhang, D. (2018). Retrospect and Prospect of Research on Frequency Regulation Technol-Ogy of Power System by Wind Power. Power Syst. Tech. 42 (06), 1793-1803. doi:10.13335/j.10003673.pst.2018.0359

Zhao, Jingjing., Min, L. I., and He, Xinqin. (2019). Coordinated Control Strategy of Wind Power and Energy Storage in Frequency Regulation Based on Torque Limit Control. Trans. China Electrotechnical Soc. 34 (23), 4982-4990.

Zheng, Zhong., Yang, Zhenyong., and Li, Weihua. (2017). Analysis and Comparison of Primary Frequency Control Technology for Wind Power and thermal Power Unit. Electric Power Automation Equipment 37 (12), 92-101. doi:10.16081/j.issn.1006-6047.2017.12.013
Conflict of Interest: Author ZZ was employed by the company Wenzhou Power Supply Company, State Grid Zhejiang Electric Power Company. Author YM was employed by the company Zaozhuang Power Supply Company, State Grid Shandong Electric Power Company. Author JZ was employed by the company Jilin Province Electric Power Company of National Grid.

The remaining authors declare that the research was conducted in the absence of any commercial or financial relationships that could be construed as a potential conflict of interest.

Publisher's Note: All claims expressed in this article are solely those of the authors and do not necessarily represent those of their affiliated organizations, or those of the publisher, the editors and the reviewers. Any product that may be evaluated in this article, or claim that may be made by its manufacturer, is not guaranteed or endorsed by the publisher.

Copyright (c) $2021 \mathrm{Li}$, Zhang, Li, Ma and Zou. This is an open-access article distributed under the terms of the Creative Commons Attribution License (CC $B Y)$. The use, distribution or reproduction in other forums is permitted, provided the original author(s) and the copyright owner(s) are credited and that the original publication in this journal is cited, in accordance with accepted academic practice. No use, distribution or reproduction is permitted which does not comply with these terms. 